\title{
Intraoperative perception and estimates on extent of resection during awake glioma surgery: overcoming the learning curve
}

\author{
Darryl Lau, MD, ${ }^{1}$ Shawn L. Hervey-Jumper, MD, ${ }^{2}$ Seunggu J. Han, MD, ${ }^{1}$ and Mitchel S. Berger, MD1 \\ 1Department of Neurological Surgery, University of California, San Francisco, California; and 'Department of Neurosurgery, \\ University of Michigan, Ann Arbor, Michigan
}

OBJECTIVE There is ample evidence that extent of resection (EOR) is associated with improved outcomes for glioma surgery. However, it is often difficult to accurately estimate EOR intraoperatively, and surgeon accuracy has yet to be reviewed. In this study, the authors quantitatively assessed the accuracy of intraoperative perception of EOR during awake craniotomy for tumor resection.

METHODS A single-surgeon experience of performing awake craniotomies for tumor resection over a 17-year period was examined. Retrospective review of operative reports for quantitative estimation of EOR was recorded. Definitive EOR was based on postoperative MRI. Analysis of accuracy of EOR estimation was examined both as a general outcome (gross-total resection [GTR] or subtotal resection [STR]), and quantitatively ( $5 \%$ within EOR on postoperative MRI). Patient demographics, tumor characteristics, and surgeon experience were examined. The effects of accuracy on motor and language outcomes were assessed.

RESULTS A total of 451 patients were included in the study. Overall accuracy of intraoperative perception of whether GTR or STR was achieved was $79.6 \%$, and overall accuracy of quantitative perception of resection (within $5 \%$ of postoperative MRI) was $81.4 \%$. There was a significant difference $(p=0.049)$ in accuracy for gross perception over the 17year period, with improvement over the later years: 1997-2000 (72.6\%), 2001-2004 (78.5\%), 2005-2008 (80.7\%), and 2009-2013 (84.4\%). Similarly, there was a significant improvement $(p=0.015)$ in accuracy of quantitative perception of EOR over the 17-year period: 1997-2000 (72.2\%), 2001-2004 (69.8\%), 2005-2008 (84.8\%), and 2009-2013 (93.4\%). This improvement in accuracy is demonstrated by the significantly higher odds of correctly estimating quantitative EOR in the later years of the series on multivariate logistic regression. Insular tumors were associated with the highest accuracy of gross perception (89.3\%; $p=0.034)$, but lowest accuracy of quantitative perception $(61.1 \%$ correct; $p<0.001)$ compared with tumors in other locations. Even after adjusting for surgeon experience, this particular trend for insular tumors remained true. The absence of $1 p 19 q$ co-deletion was associated with higher quantitative perception accuracy $(96.9 \%$ vs $81.5 \% ; p=0.051)$. Tumor grade, recurrence, diagnosis, and isocitrate dehydrogenase-1 (IDH-1) status were not associated with accurate perception of EOR. Overall, new neurological deficits occurred in $8.4 \%$ of cases, and $42.1 \%$ of those new neurological deficits persisted after the 3-month follow-up. Correct quantitative perception was associated with lower postoperative motor deficits $(2.4 \%)$ compared with incorrect perceptions $(8.0 \% ; p=0.029)$. There were no detectable differences in language outcomes based on perception of EOR.

CONCLUSIONS The findings from this study suggest that there is a learning curve associated with the ability to accurately assess intraoperative EOR during glioma surgery, and it may take more than a decade to be truly proficient. Understanding the factors associated with this ability to accurately assess EOR will provide safer surgeries while maximizing tumor resection.

https://thejns.org/doi/abs/10.3171/2017.1.JNS161811

KEY WORDS awake surgery; brain tumor; extent of resection; glioma; learning curve; mapping; oncology

ABBREVIATIONS ALA = aminolevulinic acid; $\mathrm{Cl}=$ confidence interval; $\mathrm{EOR}$ = extent of resection; IDH = isocitrate dehydrogenase; iMRI = intraoperative MRI; OR = odds ratio; OS = overall survival; PFS = progression-free survival.

SUBMITTED July 10, 2016. ACCEPTED January 19, 2017.

INCLUDE WHEN CITING Published online July 21, 2017; DOI: 10.3171/2017.1.JNS161811. 
$\mathrm{G}$ LIOMAS of WHO Grade II and higher are defined by their diffuse infiltrative nature. It is often technically challenging to discern between tumor and normal brain during surgery, especially at the tumor boundaries where clear margins are absent. ${ }^{1,2}$ Over the past decade there has been an accumulation of evidence from multiple studies showing that greater extent of resection (EOR) results in improved progression-free survival (PFS) and overall survival (OS) in both low- and high-grade gliomas. ${ }^{3,8,12,13,18}$ Therefore, there is a general consensus that achieving the greatest EOR or gross-total resection (GTR) should be the goal of surgery for intracranial gliomas. To enhance the ability to obtain greater EOR, several technological advances have been explored and deemed beneficial for the treatment of gliomas: 5-aminolevulinic acid (ALA) fluorescence, ${ }^{19}$ intraoperative MRI (iMRI), ${ }^{15}$ and neuronavigation. ${ }^{5}$ During glioma surgery, it is also imperative to preserve neurological function and avoid eloquent cortex. Awake craniotomy with cortical and subcortical (language and motor) mapping has proven to be a useful surgical approach to help identify and preserve functional areas during tumor resection. ${ }^{7}$ In addition, morbidity associated with awake craniotomies is low, especially when used in the setting of an experienced team.?

Even with the advancements that help achieve a maximal safe glioma resection, neurosurgeons still primarily rely on clinical acumen, in-depth understanding of cerebral anatomy, tactile feedback, and gross visualization of the tumor. ${ }^{2}$ These skills and competencies are critical in performing safe maximal resection of intracranial gliomas, especially when tumors are located near or within functional brain tissue. The ability to accurately and consistently estimate EOR intraoperatively may be highly beneficial, but comes with experience through cumulative operative time and case volume. There has yet to be an objective study evaluating the intraoperative ability and accuracy of the surgeon's perception in estimating EOR during glioma surgery. Therefore, in this study, we used a single neurosurgeon's experience over a 17-year period to assess the accuracy of intraoperative perception of EOR during awake glioma surgery. In addition, we describe and define the learning curve associated with this acquired skill.

\section{Methods}

\section{Patient Population}

All adult patients ages 18 years and older who underwent an awake craniotomy with functional language and/ or motor mapping for resection of a supratentorial glioma by the senior author (M.S.B.) were identified. The decision to use awake motor and/or speech mapping was conditioned on the fact that preoperative workup and imaging characteristics were most supportive of the diagnosis of an infiltrative glioma in eloquent cortex. Patients with a pathology diagnosis other than glioma were excluded from the study. The time period of interest for this study was from June 1997 to June 2013 (17-year period). All cases included preoperative imaging that involved MRI of the brain with and without gadolinium, and all cases used neuronavigation. Diffusion tensor imaging tractography for white matter pathways was available as well. Specific patient selection, anesthetic technique, and operative approach to awake craniotomy for tumor resection with cortical and subcortical functional mapping is described in detail in a prior study. ${ }^{7}$ Neuronavigation was used in all cases, and cases in which fluorescence guidance (i.e., ALA) was used to aid tumor resection or had the potential to confound the perception of tumor resection were excluded from the study. Ultrasonography was not used to guide resection in any cases. This study was approved by the Committee on Human Research of the University of California, San Francisco.

\section{Data Collection}

The primary outcome measure of interest was to evaluate the accuracy of intraoperative perception of EOR during glioma surgery. As part of the standard operative note, the senior author (M.S.B.) consistently dictates his intraoperative perception of EOR. EOR was quantified in terms of percentage tumor resected. In a few instances, only gross perception of EOR (subtotal resection [STR] vs GTR) was available. The precision of the intraoperative quantitative estimate of resection was reported as either to the single-digit percentile or, more commonly, rounded to the nearest 5th percentile. A retrospective review of the medical records was performed and these data were collected as a bivariate outcome (STR vs GTR) and quantitative continuous outcome (\%). These data were then compared with quantitative, volumetric EOR measurements between preoperative and postoperative MR images. Immediate postoperative MR images were obtained within 48 hours after surgery. Low- and high-grade tumors were volumetrically analyzed by measuring hyperintense regions on axial T2-weighted FLAIR images for low-grade tumors (Grades I and II), and on T1-weighted contrast-enhanced MR images for high-grade tumors (Grades III and IV). For each case, the tumor was segmented manually across all slices with region-of-interest analysis to compute pre- and postoperative volumes in cubic centimeters. A formally trained and experienced research associate specializing in volumetric measurement of brain tumors performed the radiological assessments, and they were blinded to the clinical, operative, and radiology reports. The EOR was calculated as follows: [100 - (postoperative tumor volume/preoperative tumor volume $) \times 100] .{ }^{13} \mathrm{De}-$ termination of tumor volume and EOR was made without consideration of the clinical outcome.

Patient demographics such as age and sex were collected. Diagnosis and tumor characteristics were also collected: histological diagnosis (glioblastoma, astrocytoma, oligoastrocytoma, oligodendroglioma, ganglioglioma, and other), WHO grade (categorized as high or low), recurrence, tumor location (frontal, temporal, insular, or parietal), cerebral hemisphere (left or right), isocitrate dehydrogenase (IDH)-1 mutation status, 1p19q status, and MIB-1 content (measured in percentage). Histopathological studies were performed on a case-by-case basis. Given the potential that inaccurate assessment of EOR could lead to continuing resection beyond the margin of tumor with subsequent injury to eloquent tissue, the secondary outcome of interest was to examine whether the accuracy of intraoperative 
perception of EOR was associated with new postoperative neurological deficit. New neurological deficit was defined as worsened neurological examination compared with preoperative baseline, which continued to persist at the time of discharge.

\section{Statistical Analysis}

Gross perception of EOR was defined as either GTR or STR, and quantitative perception of resection was the absolute percentage reported in the operative note. Accuracy was calculated by comparing intraoperative perception with postoperative MRI measurement. The threshold for a correct quantitative perception of EOR was set a priori to be within $\pm 5 \%$ of the measured EOR on postoperative MRI. Gross and quantitative perception accuracy were calculated using the following equation: (number of correct perceptions/number of total number of patients) $x$ 100. Accuracy was then stratified and evaluated by year of experience. Bivariate statistics were used to test associations between covariates and accuracy of EOR perception utilizing the chi-square test. Multivariate logistic regression was used to test associations of neurosurgeon years of experience; significant covariates were included in the multivariate model. Furthermore, a subgroup analysis based on year (1997-2000, 2001-2004, 2005-2008, and 2009-2013) was performed with chi-square analysis. The association between accuracy of EOR perception and new neurological deficit was assessed with the chi-square test. The outcomes were analyzed as overall neurological deficit (language and motor), language deficit, and motor deficit. A p value of 0.05 was used as the threshold of statistical significance. All statistical analysis was performed with SAS (version 9.3, SAS Institute).

\section{Results}

Over a 17 -year period, a total of 546 awake craniotomies for tumor resection with motor and/or language mapping were performed by the senior author (M.S.B.) at a single institution (University of California, San Francisco). Among those, 479 cases had available data regarding gross perception of resection and 425 cases had available data regarding quantitative perception of resection; 28 of those cases were excluded for nonglioma pathology. The final cohort for analysis resulted in 451 patients evaluated for gross perception of resection and 403 patients for quantitative perception of resection (Table 1). The mean age of the cohort was 42.3 years, and $60.3 \%$ were male. The distributions of diagnoses were glioblastoma in $26.2 \%$, astrocytoma in $33.5 \%$, oligoastrocytoma in $17.3 \%$, oligodendroglioma in $19.3 \%$, and ganglioglioma in $3.8 \%$. Among patients with gliomas, $47.7 \%$ had low-grade tumors (WHO Grade I or II) and $52.3 \%$ had high-grade tumors (WHO Grade III or IV). Of the 451 patients, $30.6 \%$ underwent surgery for recurrent tumors. The majority of tumors were located in the left hemisphere (94.7\%), and the distribution of tumor locations were as follows: frontal lobe (27.5\%), temporal lobe (37.5\%), insular lobe (22.8\%), and parietal lobe $(12.2 \%)$.

Table 1 shows the calculated accuracy of gross and quantitative perception of EOR. Overall accuracy of intraoperative gross perception of EOR was $79.6 \%$ (i.e., the neurosurgeon was correct that either STR or GTR was achieved in $79.6 \%$ of cases). The overall accuracy of quantitative perception of EOR within 5\% of postoperative MRI occurred in $81.4 \%$ of cases. Table 1 also shows the results of bivariate analysis testing the relationship between covariates and the accuracy of perception of EOR. Surgeon experience was significantly associated with gross perception accuracy $(\mathrm{p}=0.049)$ and quantitative perception accuracy $(\mathrm{p}=0.015)$. Accuracy of gross perception significantly improved from $70.0 \%$ to $85.7 \%$ accuracy over the 1997-2013 time period (Fig. 1). Subgroups based on 4 time periods also showed improvement in accuracy $(\mathrm{p}=0.175):$ 1997-2000 (72.6\%), 2001-2004 (78.5\%), 2005-2008 (80.7\%), and 2009-2013 (84.4\%). Similarly, accuracy of quantitative perception significantly improved from $77.8 \%$ to $100.0 \%$ over the 17-year time period (Fig. 2). Subgroup analysis showed significant improvement as well (p < 0.001): 1997-2000 (72.2\%), 2001-2004 (69.8\%), 2005-2008 (84.8\%), and 2009-2013 (93.4\%). Tumor location was also significantly associated with perception accuracy. For gross perception accuracy, tumors located in the insula had significantly higher accuracy (89.3\%) compared with tumors located in other locations: frontal $(74.2 \%)$, temporal $(77.5 \%)$, and parietal $(80.0 \%$; $p=0.034)$. However, the accuracy of quantitative perception of EOR for tumors in the insular lobe was significantly lower than that compared with tumors in other locations $(\mathrm{p}<0.001)$. Quantitative perception accuracy for insular tumors was $61.1 \%$, while for frontal, temporal, and parietal tumors accuracies were $87.8 \%, 86.8 \%$, and $87.0 \%$, respectively.

Multivariate logistic regression analysis was performed to test the relationship of surgical experience to the neurosurgeon's intraoperative perception of gross and quantitative EOR adjusted by tumor location (Table 2). Relative to an earlier year in the series (2002), there were significantly higher odds of estimating a correct quantitative EOR in the following years: 2005 (odds ratio [OR] 13.07, 95\% confidence interval [CI] 1.41-120.86, $\mathrm{p}=0.024), 2007$ (OR $6.21,95 \%$ CI 1.36-28.44, $\mathrm{p}=0.019$ ), 2009 (OR 11.72, 95\% CI $2.07-66.45, \mathrm{p}=0.005$ ), 2010 (OR 7.60, 95\% CI $1.37-$ 42.07, $\mathrm{p}=0.020$ ), 2011 (OR 22.73, 95\% CI 2.49-207.68, $\mathrm{p}=0.006$ ), and 2012 (OR 5.93, 95\% CI 1.30-27.01, $\mathrm{p}=$ 0.022 ). Insular location was independently associated with higher odds of correct gross perception (OR 3.45, 95\% CI $1.58-7.49, \mathrm{p}=0.002$ ) and lower odds of correct quantitative perception (OR 0.18, 95\% CI 0.08-0.39, p < 0.001).

Table 3 shows the results of testing associations between histological characteristics and perception of resection. IDH-1 mutation status was not associated with differences in gross $(p=0.431)$ or quantitative perception $(p=0.562)$ accuracy. Similarly, there was no significant difference in gross perception accuracy based on $1 \mathrm{p} 19 \mathrm{q}$ deletion status $(\mathrm{p}=0.567)$ or MIB-1 $(\mathrm{p}=0.876)$. The absence of a $1 \mathrm{p} 19 \mathrm{q}$ deletion was associated with higher quantitative perception accuracy, but this was only close to being significant (96.9\% vs $81.5 \%$; $p=0.051)$. There was a nonsignificant trend for greater accuracy of quantitative perception with larger MIB-1 indices: < 5\% (78.9\%), 5\%-15\% (82.6\%), and $>15 \%(91.9 \% ; \mathrm{p}=0.200)$. 
TABLE 1. Factors associated with surgeon's intraoperative perception of gross-total and quantitative EOR

\begin{tabular}{|c|c|c|c|c|c|c|c|c|c|c|}
\hline \multirow[b]{3}{*}{ Variable } & \multicolumn{5}{|c|}{ Gross Perception } & \multicolumn{5}{|c|}{ Quantitative Perception } \\
\hline & \multicolumn{2}{|l|}{ Overall } & \multicolumn{3}{|c|}{ Accuracy } & \multicolumn{2}{|c|}{ Overall } & \multicolumn{3}{|c|}{ Accuracy } \\
\hline & No. of Cases & $\%$ & No. of Cases & $\%$ & $p$ Value & No. of Cases & $\%$ & No. of Cases & $\%$ & $p$ Value \\
\hline Overall & 451 & - & 359 & 79.6 & & 403 & - & 328 & 81.4 & \\
\hline Surgeon experience & & & & & 0.049 & & & & & 0.015 \\
\hline 1997 & 10 & 2.2 & 7 & 70.0 & & 9 & 2.2 & 7 & 77.8 & \\
\hline 1998 & 19 & 4.2 & 12 & 63.2 & & 18 & 4.5 & 13 & 72.2 & \\
\hline 1999 & 34 & 7.5 & 26 & 76.5 & & 33 & 8.2 & 24 & 72.7 & \\
\hline 2000 & 32 & 7.1 & 24 & 75.0 & & 30 & 7.4 & 21 & 70.0 & \\
\hline 2001 & 24 & 5.3 & 20 & 83.3 & & 18 & 4.5 & 13 & 72.2 & \\
\hline 2002 & 27 & 6.0 & 19 & 70.4 & & 24 & 6.0 & 15 & 62.5 & \\
\hline 2003 & 20 & 4.4 & 19 & 95.0 & & 15 & 3.7 & 11 & 73.3 & \\
\hline 2004 & 36 & 8.0 & 26 & 72.2 & & 29 & 7.2 & 21 & 72.4 & \\
\hline 2005 & 23 & 5.1 & 19 & 82.6 & & 21 & 5.2 & 20 & 95.2 & \\
\hline 2006 & 32 & 7.1 & 27 & 84.4 & & 27 & 6.7 & 21 & 77.8 & \\
\hline 2007 & 29 & 6.4 & 26 & 89.7 & & 29 & 7.2 & 26 & 89.7 & \\
\hline 2008 & 30 & 6.7 & 20 & 66.7 & & 28 & 6.9 & 22 & 78.6 & \\
\hline 2009 & 28 & 6.2 & 24 & 85.7 & & 27 & 6.7 & 25 & 92.6 & \\
\hline 2010 & 30 & 6.7 & 25 & 83.3 & & 28 & 6.9 & 26 & 92.9 & \\
\hline 2011 & 32 & 7.1 & 27 & 84.4 & & 29 & 7.2 & 28 & 96.6 & \\
\hline 2012 & 38 & 8.4 & 32 & 84.2 & & 32 & 7.9 & 29 & 90.6 & \\
\hline 2013 & 7 & 1.6 & 6 & 85.7 & & 6 & 1.5 & 6 & 100.0 & \\
\hline Age (yrs) & & & & & 0.641 & & & & & 0.483 \\
\hline$<45$ & 276 & 61.2 & 222 & 80.4 & & 245 & 60.8 & 200 & 81.6 & \\
\hline $45-65$ & 150 & 33.3 & 116 & 77.3 & & 137 & 34.0 & 109 & 79.6 & \\
\hline$>65$ & 25 & 5.5 & 21 & 84.0 & & 21 & 5.2 & 19 & 90.5 & \\
\hline Sex & & & & & 0.401 & & & & & 0.243 \\
\hline Male & 179 & 39.7 & 146 & 81.6 & & 164 & 40.7 & 129 & 78.7 & \\
\hline Female & 272 & 60.3 & 213 & 78.3 & & 239 & 59.3 & 199 & 83.3 & \\
\hline Diagnosis & & & & & 0.437 & & & & & 0.318 \\
\hline Glioblastoma & 118 & 26.2 & 87 & 73.7 & & 111 & 27.5 & 96 & 86.5 & \\
\hline Astrocytoma & 151 & 33.5 & 122 & 80.8 & & 131 & 32.5 & 100 & 76.3 & \\
\hline Oligoastrocytoma & 78 & 17.3 & 63 & 80.8 & & 66 & 16.4 & 53 & 80.3 & \\
\hline Oligodendroglioma & 87 & 19.3 & 73 & 83.9 & & 78 & 19.4 & 64 & 82.1 & \\
\hline Ganglioglioma & 17 & 3.8 & 14 & 82.4 & & 17 & 4.2 & 15 & 88.2 & \\
\hline Grade & & & & & 0.504 & & & & & 0.095 \\
\hline Low (I or II) & 215 & 47.7 & 174 & 80.9 & & 196 & 48.6 & 153 & 78.1 & \\
\hline High (III or IV) & 236 & 52.3 & 185 & 78.4 & & 207 & 51.4 & 175 & 84.5 & \\
\hline Recurrence & & & & & 0.329 & & & & & 0.207 \\
\hline Yes & 138 & 30.6 & 106 & 76.8 & & 121 & 30.0 & 103 & 85.1 & \\
\hline No & 313 & 69.4 & 253 & 80.8 & & 282 & 70.0 & 225 & 79.8 & \\
\hline Location & & & & & 0.034 & & & & & $<0.001$ \\
\hline Frontal & 124 & 27.5 & 92 & 74.2 & & 115 & 28.5 & 101 & 87.8 & \\
\hline Temporal & 169 & 37.5 & 131 & 77.5 & & 152 & 37.7 & 132 & 86.8 & \\
\hline Insular & 103 & 22.8 & 92 & 89.3 & & 90 & 22.3 & 55 & 61.1 & \\
\hline Parietal & 55 & 12.2 & 44 & 80.0 & & 46 & 11.4 & 40 & 87.0 & \\
\hline Hemisphere & & & & & 0.565 & & & & & 0.601 \\
\hline $\mathrm{Lt}$ & 427 & 94.7 & 341 & 79.9 & & 382 & 94.8 & 310 & 81.2 & \\
\hline $\mathrm{Rt}$ & 24 & 5.3 & 18 & 75.0 & & 21 & 5.2 & 18 & 85.7 & \\
\hline
\end{tabular}




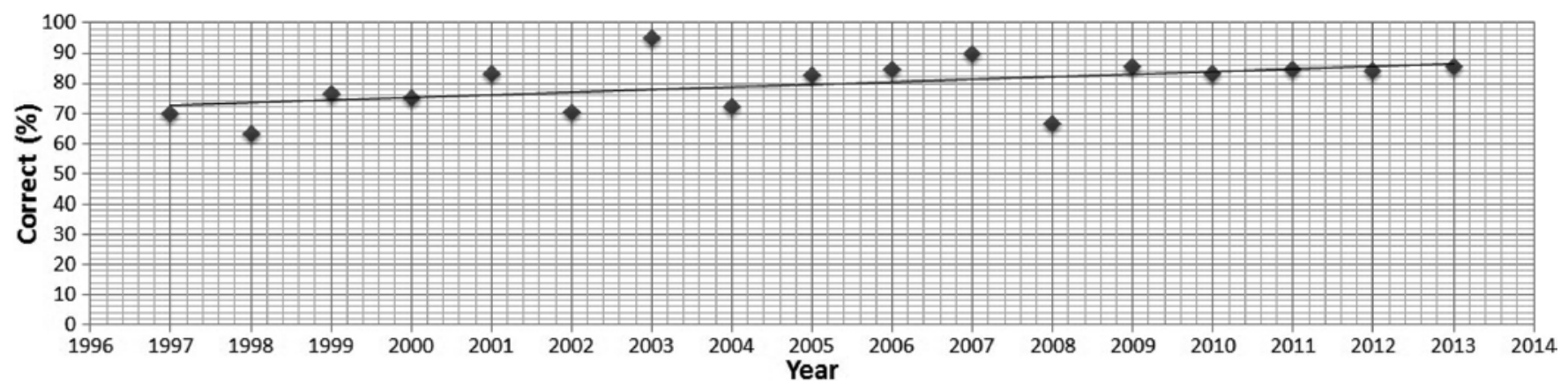

FIG. 1. Line graph of gross perception of EOR for awake glioma surgery. Gross perception was defined as whether GTR or STR was achieved compared with postoperative MRI measurements. Over the course of 17 years, there was a significant trend for improvement in accuracy of gross perception from $70.0 \%$ to $85.7 \%(p=0.049)$. Subgroups based on 4 time periods also showed improvement in accuracy: 1997-2000 (72.6\%), 2001-2004 (78.5\%), 2005-2008 (80.7\%), and 2009-2013 (84.4\%; $p=0.175)$.

Tables 4 and 5 show the analysis between accuracy of gross and quantitative perception of EOR and its association with new postoperative neurological deficits. Overall new neurological deficits occurred in $8.4 \%$ of cases, and $42.1 \%$ of those new postoperative neurological deficits persisted after the 3-month follow-up. In regard to gross perception of EOR, there was no significant association between accuracy and neurological deficit $(\mathrm{p}=0.674)$, even when stratified by language $(\mathrm{p}=0.438)$ or motor $(\mathrm{p}$ $=0.520$ ) deficits. For quantitative perception, there was a trend for correct estimates to have lower rates of neurological deficits, but this was not significant $(\mathrm{p}=0.184)$. However, when stratified by type of neurological deficit, correct quantitative perceptions were associated with significantly lower rates of motor deficits $(2.4 \%)$ compared with incorrect perceptions $(8.0 \% ; \mathrm{p}=0.029)$. There was no significant difference in the rate of language deficits when comparing incorrect and correct perception $(6.7 \%$ vs $5.2 \%$, respectively; $\mathrm{p}=0.578$ ).

\section{Discussion}

The goals of glioma surgery are to obtain maximal resection or GTR while preserving neurological function, and often there is a fine balance between these two goals. To perform this type of surgery, intraoperative decisions on when to stop resecting tumor is critical. However, this can be quite challenging due to the infiltrative nature of gliomas, resulting in difficulty distinguishing a clear margin between tumor and normal brain. This translates into obscurity when estimating how much tumor is removed intraoperatively. Being able to accurately assess EOR is an essential skill that may mitigate neurological deficit and morbidity, especially for tumors in eloquent locations. The findings from this study, which suggest that improved functional outcomes may be associated with more accurate assessment of EOR, further support the value of this skill.

To date, there are no studies examining a neurosurgeon's ability to estimate intraoperative EOR during glioma surgery. Therefore, this study seeks to define the learning curve associated with this skill and identify tumor characteristics that influence the perception of resection during awake craniotomy for resection of eloquent tumors. This study's results suggest cumulative surgical experience has a significant influence in the ability to intraoperatively estimate EOR grossly and quantitatively; there was a linear trend for greater accuracy with more years of surgical experience. Other factors identified that were significantly associated with greater difficulty of estimating EOR included tumors located in the insula and possibly tumors harboring $1 \mathrm{p} 19 \mathrm{q}$ deletions. There was a nonsignificant trend for low-grade tumors to be associated with less accurate estimates of EOR. In addition, findings from this study suggest that incorrect quantitative perception of resection is associated with higher rates of postoperative motor deficits.

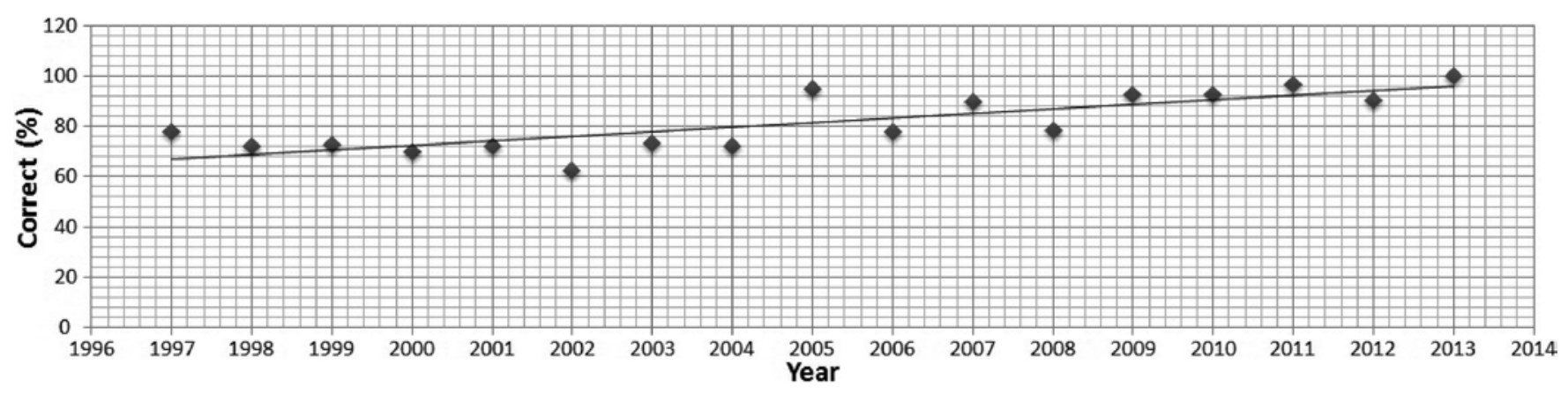

FIG. 2. Line graph of quantitative perception of EOR for awake glioma surgery. Quantitative perception was defined as whether the percentage of resection achieved was within $5 \%$ of postoperative MRI measurements. Accuracy of quantitative perception significantly improved from $77.8 \%$ to $100 \%$ over the 17 -year time period $(p=0.015)$. Subgroup analysis showed significant improvement as well: 1997-2000 (72.2\%), 2001-2004 (69.8\%), 2005-2008 (84.8\%), and 2009-2013 (93.4\%; $p<0.001)$. 
TABLE 2. Multivariate logistic regression analysis assessing the relationship between correct intraoperative assessment of EOR and surgical experience

\begin{tabular}{|c|c|c|c|c|c|c|}
\hline \multirow[b]{2}{*}{ Variable } & \multicolumn{3}{|c|}{ Gross Perception } & \multicolumn{3}{|c|}{ Quantitative Perception } \\
\hline & OR & $\mathrm{Cl}$ & $p$ Value & OR & $\mathrm{Cl}$ & p Value \\
\hline \multicolumn{7}{|c|}{ Yr of surgeon experience } \\
\hline 1997 & 1.14 & $0.21-6.19$ & 0.880 & 3.64 & $0.54-24.35$ & 0.183 \\
\hline 1998 & Ref & Ref & Ref & 1.53 & $0.38-6.14$ & 0.553 \\
\hline 1999 & 2.12 & $0.61-7.37$ & 0.238 & 1.39 & $0.42-4.55$ & 0.587 \\
\hline 2000 & 1.53 & $0.44-5.36$ & 0.510 & 2.02 & $0.59-6.91$ & 0.261 \\
\hline 2001 & 2.96 & $0.70-12.50$ & 0.141 & 1.73 & $0.43-7.00$ & 0.446 \\
\hline 2002 & 1.43 & $0.40-5.06$ & 0.584 & Ref & Ref & Ref \\
\hline 2003 & 11.53 & $1.24-107.26$ & 0.032 & 2.00 & $0.44-9.00$ & 0.367 \\
\hline 2004 & 1.22 & $0.36-4.12$ & 0.748 & 2.20 & $0.63-7.64$ & 0.214 \\
\hline 2005 & 2.83 & $0.67-12.01$ & 0.159 & 13.07 & $1.41-120.86$ & 0.024 \\
\hline 2006 & 3.33 & $0.86-12.88$ & 0.082 & 2.34 & $0.64-8.61$ & 0.202 \\
\hline 2007 & 5.22 & $1.13-24.18$ & 0.035 & 6.21 & $1.36-28.44$ & 0.019 \\
\hline 2008 & 1.17 & $0.34-3.99$ & 0.801 & 2.47 & $0.68-9.03$ & 0.172 \\
\hline 2009 & 3.30 & $0.79-13.84$ & 0.102 & 11.72 & $2.07-66.45$ & 0.005 \\
\hline 2010 & 3.19 & $0.82-12.42$ & 0.094 & 7.60 & $1.37-42.07$ & 0.020 \\
\hline 2011 & 3.12 & $0.81-12.08$ & 0.100 & 22.73 & $2.49-207.68$ & 0.006 \\
\hline 2012 & 3.19 & $0.87-11.68$ & 0.080 & 5.93 & $1.30-27.01$ & 0.022 \\
\hline 2013 & 4.10 & $0.40-42.48$ & 0.237 & $>999.99$ & $<0.01$ to $>999.99$ & 0.982 \\
\hline \multicolumn{7}{|l|}{ Glioma location } \\
\hline Frontal & Ref & Ref & Ref & Ref & Ref & Ref \\
\hline Temporal & 1.25 & $0.71-2.19$ & 0.439 & 0.86 & $0.40-1.85$ & 0.703 \\
\hline Insular & 3.45 & $1.58-7.49$ & 0.002 & 0.18 & $0.08-0.39$ & $<0.001$ \\
\hline Parietal & 1.39 & $0.62-3.12$ & 0.423 & 0.73 & $0.25-2.14$ & 0.566 \\
\hline
\end{tabular}

Ref $=$ referent

Several technological advances are now being used to aid in the identification of tumor during glioma surgery and help estimate EOR, including neuronavigation, ${ }^{5}$ iMRI ${ }^{15}$ and ALA fluorescence. ${ }^{19}$ However, there are intrinsic limitations to each modality. Neuronavigation has become nearly standard practice in the US during brain tumor surgery, and its use is straightforward. The main limitation of neuronavigation is its susceptibility to brain shift and displacement during surgery, with the cortical surfaces most affected. Shown in a prospective study by Reinges et al., even dural opening results in brain shifts, and greater displacement occurs during tumor resection (up to $1.5 \mathrm{~cm}$ ), resulting in loss of reliability of the neuronavigation system. ${ }^{11} \mathrm{~A}$ solution to this is to use iMRI, in which new imaging and neuronavigation registration can be obtained during surgery. However, this approach is not readily available to all institutions, limits the surgeon to using MRI-compatible equipment, and lengthens surgical

TABLE 3. Histological markers associated with gross and quantitative intraoperative perception of EOR

\begin{tabular}{|c|c|c|c|c|c|c|c|c|c|c|}
\hline \multirow[b]{2}{*}{ Marker } & \multicolumn{2}{|l|}{ Overall } & \multicolumn{3}{|c|}{ Gross Perception Accuracy } & \multicolumn{2}{|l|}{ Overall } & \multicolumn{3}{|c|}{ Quantitative Perception Accuracy } \\
\hline & No. of Cases & $\%$ & No. of Cases & $\%$ & $p$ Value & No. of Cases & $\%$ & No. of Cases & $\%$ & $\mathrm{p}$ Value \\
\hline IDH-1 mutation & 67 & - & 57 & 85.1 & 0.431 & 57 & - & 53 & 93.0 & 0.562 \\
\hline Yes & 41 & 61.2 & 36 & 87.8 & & 35 & 61.4 & 32 & 91.4 & \\
\hline No & 26 & 38.8 & 21 & 80.8 & & 22 & 38.6 & 21 & 95.5 & \\
\hline $1 p 19 q$ deletion & 66 & - & 58 & 87.9 & 0.567 & 59 & - & 53 & 89.8 & 0.051 \\
\hline Yes & 31 & 47.0 & 28 & 90.3 & & 27 & 45.8 & 22 & 81.5 & \\
\hline No & 35 & 53.0 & 30 & 85.7 & & 32 & 54.2 & 31 & 96.9 & \\
\hline MIB-1 & 243 & - & 201 & 82.7 & 0.876 & 220 & - & 181 & 82.3 & 0.200 \\
\hline$<5 \%$ & 124 & 51.0 & 102 & 82.3 & & 114 & 51.8 & 90 & 78.9 & \\
\hline $5 \%-15 \%$ & 77 & 31.7 & 65 & 84.4 & & 69 & 31.4 & 57 & 82.6 & \\
\hline$>15 \%$ & 42 & 17.3 & 34 & 81.0 & & 37 & 16.8 & 34 & 91.9 & \\
\hline
\end{tabular}


TABLE 4. Effect of gross perception accuracy on motor and language outcomes

\begin{tabular}{ccccccc}
\hline \multirow{2}{*}{$\begin{array}{c}\text { Gross } \\
\text { Perception }\end{array}$} & \multicolumn{2}{c}{ Overall } & & \multicolumn{3}{c}{ Neurological Deficit } \\
\cline { 2 - 3 } \cline { 6 - 7 } & No. of Cases & $\%$ & & No. of Cases & $\%$ & $\mathrm{p}$ Value \\
\hline Overall & 451 & - & & 38 & 8.4 & 0.674 \\
\hline Incorrect & 92 & 20.4 & & 9 & 9.8 & \\
\hline Correct & 359 & 79.6 & 29 & 8.1 & \\
\hline Language & 451 & - & 23 & 5.1 & 0.438 \\
\hline Incorrect & 92 & 20.4 & 6 & 6.5 & \\
\hline Correct & 359 & 79.6 & 17 & 4.7 & \\
\hline Motor & 451 & - & 15 & 3.3 & 0.520 \\
\hline Incorrect & 92 & 20.4 & 4 & 4.3 & \\
\hline Correct & 359 & 79.6 & 11 & 3.1 & \\
\hline
\end{tabular}

duration. More recently, ALA has been shown to be effective in improving GTR rates of high-grade gliomas and lengthening PFS and OS, but its use is pending approval in the US..$^{9}$ Therefore, to achieve a safe glioma resection, neurosurgeons still primarily rely on clinical acumen, indepth understanding of cerebral anatomy, tactile feedback, and gross visualization of the tumor. ${ }^{2}$

Based on the findings from this study, the accuracy in estimating EOR in glioma surgery is highly influenced by surgical experience. The results from this study parallel the findings from the general surgery and neurosurgery literature, which demonstrate that greater surgical experience and higher case volume are associated with lower morbidity and better outcomes..$^{10,14,21}$ Specifically, Trinh et al. has evaluated the effect of provider and hospital caseload on complication rates following craniotomy for resection of supratentorial brain tumors. ${ }^{21}$ In their study, surgeons with high case volume had significantly lower complications and in-hospital deaths. This is not a surprising or new concept, but no one has yet provided details and/or described how much experience is needed for brain tumor surgery. In addition, there are no available studies or data describing the neurosurgeon's ability to estimate EOR during glioma surgery. The findings from this study show a linear improvement in accuracy over the 17-year period studied (gross perception $70.0 \%-85.7 \%$ and quantitative perception $66.7 \%-100.0 \%$ ), representing the learning curve. In fact, multivariate logistic regression analysis revealed that the neurosurgeon had significantly higher odds of predicting accurate quantitative EOR in the last 4 to 5 years of the series compared with his earlier years. Experience over the years is obviously dependent on case volume as well. The neurosurgeon in this study has a high-volume tumor practice, and when including brain tumor cases not reported in this study (asleep cases), he has performed more than 3000 brain tumor surgeries in total during the study period (averaging 176 tumor surgeries/year). In addition, prior to that in an 11-year period, he has performed approximately 2200 brain tumor surgeries. These results support the notion that it is difficult to obtain competence in accurately estimating intraoperative EOR, but mastery can be achieved with experience.

Other factors associated with perception of EOR were components related to tumor location, characteristics, and
TABLE 5. Effect of quantitative perception accuracy on motor and language outcomes

\begin{tabular}{crrrrr}
\hline Quantitative & No. of & & \multicolumn{3}{c}{ Neurological Deficit } \\
\cline { 4 - 6 } Perception & Cases & $\%$ & No. of Cases & $\%$ & p Value \\
\hline Overall & 403 & - & 37 & 9.2 & 0.184 \\
\hline Incorrect & 75 & 18.6 & 10 & 13.3 & \\
\hline Correct & 328 & 81.4 & 27 & 8.2 & \\
\hline Language & 403 & - & 22 & 5.5 & 0.578 \\
\hline Incorrect & 75 & 18.6 & 5 & 6.7 & \\
\hline Correct & 328 & 81.4 & 17 & 5.2 & \\
\hline Motor & 403 & - & 14 & 3.5 & 0.029 \\
\hline Incorrect & 75 & 18.6 & 6 & 8.0 & \\
\hline Correct & 328 & 81.4 & 8 & 2.4 & \\
\hline
\end{tabular}

histological markers. Insular tumors were associated with higher rates of accurate gross perception of EOR (89.3\% vs $74.2 \%-80.0 \%$ in other lobes) but lower rates of accurate quantitative perception of EOR (61.1\% compared with $86.8 \%-87.8 \%$ in other lobes). These contradictory results are not easily understood but can be rationally explained. Tumors involving the insula are difficult to approach due to proximity of the major sylvian vessels and eloquent (motor and language) pathways. These critical structures are protected by either splitting the sylvian fissure or opening up multiple "windows" allowing an approach to the tumor at different angles. However, this leaves the neurosurgeon to work within deep corridors with only limited views of small areas within the tumor and surgical cavity. Therefore, it can be very challenging to accurately estimate the amount (quantity) of tumor present or EOR. In contrast, it is relatively straightforward to perceive whether any tumor remains (i.e., whether STR or GTR was achieved).

Lower MIB-1 indices and the presence of $1 \mathrm{p} 19 \mathrm{q}$ deletion status were associated with lower abilities to estimate EOR. MIB-1 and 1p19q status are likely histological and genetic surrogates of gross tumor morphology, thus affecting the perception and ability to identify tumor tissue through gross visualization and tactile feedback. MIB-1 is a stain used to measure mitotic and proliferative activity, with greater MIB-1 indices often correlating with higher grade and more malignant behavior. ${ }^{17}$ Higher-grade tumors, such as glioblastoma, tend to have more discoloration due to necrotic tissue and the development of microvasculature, making these tumors more obvious during gross visualization. The tactile feel during surgery is likely different compared with normal brain as well. This rationale is further supported by the observed trend that glioblastomas have the highest accuracy in quantifying EOR in this study. The association between $1 \mathrm{p} 19 \mathrm{q}$ co-deletion and the reduced ability to perceive EOR is unclear. Recently, a genome-wide analysis of gliomas demonstrated that molecular and genetic markers were more correlated with clinical outcome than histological diagnosis. ${ }^{4}$ Specifically, low-grade gliomas with IDH-1 mutations and 1p19q co-deletion have the most favorable outcome, indicating a lower grade phenotype. Gross morphology may also reflect this during resection, making it more difficult to distinguish between tumor and brain (lack of necrosis, discolor- 
ation, and microvasculature). Further studies are needed to substantiate this observation.

Awake brain mapping has become the standard approach to maximizing EOR while minimizing neurological morbidity during the resection of intracranial gliomas within or in proximity to eloquent cortex ${ }^{6,7}$ Immediate new neurological deficits and morbidity following resection of eloquent brain tumors with awake mapping ranges from $8 \%$ to $26 \%, 7,16,20$ This neurological deficit rate in our study falls into the lower range of the reported rates (8.4\%), and approximately half remain present at the 3-month follow-up. New deficits are usually related to the proximity of functional eloquent brain tissue with risk for edema and/or ischemic injury. However, even after supplementing awake craniotomy with iMRI, the deficit rates are similar, ranging from $20 \%$ to $26 \%$, further highlighting the importance of the ability to identify tumor borders., ${ }^{9,22}$ In this study, incorrect estimations of quantitative EOR were associated with a small but significantly higher rate (5.6\%) of new motor deficits. A similar trend was observed with language deficits, but the difference was small (1.7\%) and this was not significant. The hypothesis driving these specific analyses stems from the fact that infiltrative gliomas are often difficult to discern at the tumor-brain interface, and while trying to maximize EOR, inaccurate intraoperative estimates of EOR could potentially lead to continuing the resection beyond the margin of the tumor with subsequent potential for injury to eloquent tissue. It is unclear why the motor pathways are more susceptible than the language pathways when the tumor margins are inaccurately estimated, but this could be related to the difference in testing methods for each function and/or differences in anatomical organization of these language and motor pathways. Nonetheless, the ability to estimate EOR accurately appears to have clinical significance and potential benefit for patient outcome.

There are limitations related to the retrospective nature of the study design. One of these limitations is the susceptibility of missing data points from a retrospective review of medical records. However, the majority of data points were available in this study and the cohort remains relatively large. Second, retrospective studies and post hoc subgroup analyses are innately vulnerable to confounders and unforeseen associated factors that may affect the outcome of interest. As the goal of this study was to evaluate the surgeon's ability to accurately predict the EOR and although all cases used neuronavigation, it is plausible that technological advancements in imaging and operative equipment may account for some of the findings from this study. However, this is less likely given that technological advances in imaging should also lead to greater ability to detect tumor on postoperative imaging. One of the strengths of this study is the utilization of a single surgeon's experience and avoidance of variability. Future studies should consider utilizing multiple surgeons to compare the perception of tumor resection among brain tumor surgeons in different stages of their careers.

\section{Conclusions}

It is often difficult to differentiate tumor border from normal brain because of the infiltrative nature of gliomas.
It is important to understand the learning curve and factors associated with being able to accurately assess EOR. The findings from this study suggest that there is a learning curve associated with being able to accurately estimate intraoperative EOR during glioma surgery, and it may take more than a decade and thousands of cases to become truly proficient. Tumor location and morphology are factors that have the most influence on the surgeon's perception of EOR; insular tumors and tumors with 1p19q deletion are the most difficult for quantitatively estimating the EOR. There also appears to be a reduction in neurological morbidity when surgeon perception is more accurate. This study illustrates that accurately assessing EOR intraoperatively is a valuable skill set that develops over a neurosurgeon's career. While accuracy of assessing EOR comes with experience, it may be advantageous for neurosurgical mentors to make a conscious effort to teach this particular skill to trainees. Additional studies of this topic are warranted in the future.

\section{References}

1. Albert FK, Forsting M, Sartor K, Adams HP, Kunze S: Early postoperative magnetic resonance imaging after resection of malignant glioma: objective evaluation of residual tumor and its influence on regrowth and prognosis. Neurosurgery 34:45-61, 1994

2. Berger MS: Glioma surgery: a century of challenge. Clin Neurosurg 58:7-9, 2011

3. Bloch O, Han SJ, Cha S, Sun MZ, Aghi MK, McDermott MW, et al: Impact of extent of resection for recurrent glioblastoma on overall survival: clinical article. J Neurosurg 117:1032-1038, 2012

4. Brat DJ, Verhaak RG, Aldape KD, Yung WK, Salama SR, Cooper LA, et al: Comprehensive, integrative genomic analysis of diffuse lower-grade gliomas. N Engl J Med 372:24812498, 2015

5. D’Amico RS, Kennedy BC, Bruce JN: Neurosurgical oncology: advances in operative technologies and adjuncts. J Neurooncol 119:451-463, 2014

6. Freyschlag CF, Duffau H: Awake brain mapping of cortex and subcortical pathways in brain tumor surgery. J Neurosurg Sci 58:199-213, 2014

7. Hervey-Jumper SL, Li J, Lau D, Molinaro AM, Perry DW, Meng L, et al: Awake craniotomy to maximize glioma resection: methods and technical nuances over a 27 -year period. J Neurosurg 123:325-339, 2015

8. Ius T, Isola M, Budai R, Pauletto G, Tomasino B, Fadiga L, et al: Low-grade glioma surgery in eloquent areas: volumetric analysis of extent of resection and its impact on overall survival. A single-institution experience in 190 patients: clinical article. J Neurosurg 117:1039-1052, 2012

9. Maldaun MV, Khawja SN, Levine NB, Rao G, Lang FF, Weinberg JS, et al: Awake craniotomy for gliomas in a highfield intraoperative magnetic resonance imaging suite: analysis of 42 cases. J Neurosurg 121:810-817, 2014

10. McAteer JP, LaRiviere CA, Drugas GT, Abdullah F, Oldham $\mathrm{KT}$, Goldin AB: Influence of surgeon experience, hospital volume, and specialty designation on outcomes in pediatric surgery: a systematic review. JAMA Pediatr 167:468-475, 2013

11. Reinges MH, Nguyen HH, Krings T, Hütter BO, Rohde V, Gilsbach JM: Course of brain shift during microsurgical resection of supratentorial cerebral lesions: limits of conventional neuronavigation. Acta Neurochir (Wien) 146:369377,2004

12. Sanai N, Berger MS: Glioma extent of resection and its im- 
pact on patient outcome. Neurosurgery 62:753-764, 264266, 2008

13. Sanai N, Polley MY, McDermott MW, Parsa AT, Berger MS: An extent of resection threshold for newly diagnosed glioblastomas. J Neurosurg 115:3-8, 2011

14. Schmidt CM, Turrini O, Parikh P, House MG, Zyromski NJ, Nakeeb A, et al: Effect of hospital volume, surgeon experience, and surgeon volume on patient outcomes after pancreaticoduodenectomy: a single-institution experience. Arch Surg 145:634-640, 2010

15. Senft C, Bink A, Franz K, Vatter H, Gasser T, Seifert V: Intraoperative MRI guidance and extent of resection in glioma surgery: a randomised, controlled trial. Lancet Oncol 12:997-1003, 2011

16. Shinoura N, Midorikawa A, Yamada R, Hana T, Saito A, Hiromitsu K, et al: Awake craniotomy for brain lesions within and near the primary motor area: A retrospective analysis of factors associated with worsened paresis in 102 consecutive patients. Surg Neurol Int 4:149, 2013

17. Skjulsvik AJ, Mørk JN, Torp MO, Torp SH: Ki-67/MIB-1 immunostaining in a cohort of human gliomas. Int J Clin Exp Pathol 7:8905-8910, 2014

18. Smith JS, Chang EF, Lamborn KR, Chang SM, Prados MD, Cha S, et al: Role of extent of resection in the long-term outcome of low-grade hemispheric gliomas. J Clin Oncol 26:1338-1345, 2008

19. Stummer W, Pichlmeier U, Meinel T, Wiestler OD, Zanella F, Reulen HJ: Fluorescence-guided surgery with 5-aminolevulinic acid for resection of malignant glioma: a randomised controlled multicentre phase III trial. Lancet Oncol 7:392401, 2006

20. Trimble G, McStravick C, Farling P, Megaw K, McKinstry $\mathrm{S}$, Smyth G, et al: Awake craniotomy for glioma resection:
Technical aspects and initial results in a single institution. $\mathbf{B r}$ J Neurosurg 29:836-842, 2015

21. Trinh VT, Davies JM, Berger MS: Surgery for primary supratentorial brain tumors in the United States, 2000-2009: effect of provider and hospital caseload on complication rates. J Neurosurg 122:280-296, 2015

22. Tuominen J, Yrjänä S, Ukkonen A, Koivukangas J: Awake craniotomy may further improve neurological outcome of intraoperative MRI-guided brain tumor surgery. Acta Neurochir (Wien) 155:1805-1812, 2013

\section{Disclosures}

The authors report no conflict of interest concerning the materials or methods used in this study or the findings specified in this paper.

\section{Author Contributions}

Conception and design: Berger, Lau. Acquisition of data: Lau, Hervey-Jumper. Analysis and interpretation of data: Lau, HerveyJumper, Han. Drafting the article: Lau. Critically revising the article: all authors. Reviewed submitted version of manuscript: all authors. Approved the final version of the manuscript on behalf of all authors: Berger. Statistical analysis: Lau. Study supervision: Berger.

\section{Correspondence}

Mitchel S. Berger, Department of Neurological Surgery, University of California, San Francisco, 505 Parnassus Ave., M779, San Francisco, CA 94143. email: bergerm@neurosurg.ucsf.edu. 